\title{
Der neue Lohnausweis
}

\author{
Auf den 1. Januar 2007 wird der neue Lohnausweis (NLA) verbindlich einge- \\ führt. Der NLA ist die wichtigste Grundlage für die Besteuerung des Arbeit- \\ nehmers und für die Abzugsmöglichkeiten bei den Berufsauslagen jedes \\ einzelnen.
}

Guido Schmid

Korrespondenz:

Guido Schmid, dipl. Treuhandexperte ABT Treuhandgesellschaft Andreas Baumann und Co. FMH Treuhand Services Alte Steinhauserstrasse 1 CH-6330 Cham Tel. 0417486290 Fax 0417486201 guido.schmid@fmhtreuhand.ch
Das Ziel des NLA besteht hauptsächlich darin, durch ein schweizweit einheitliches Formular mehr Transparenz und Rechtsgleichheit zu schaffen. Es gibt bisher mehrere unterschiedliche Formulare. Die entsprechenden Erläuterungen, die vor Jahren erarbeitet wurden, äusserten sich nicht zu den modernen Gehaltsleistungen und Gehaltsnebenleistungen. Die Arbeitgeber nehmen ihre Deklarationspflicht oft uneinheitlich vor.

Der NLA führt auch dazu, dass Leistungen, die bisher nicht oder teilweise besteuert wurden, neu der Besteuerung unterliegen.

Beim Übergang zum NLA könnte erkannt werden, dass in der Vergangenheit gewisse Lohnausweise unvollständig ausgefüllt worden sind. Werden Fehler in Lohnausweisen früherer Jahre festgestellt und war es nicht die Absicht, dadurch fahrlässig oder vorsätzlich Steuerhinterziehung zu ermöglichen, ist ein angemessenes, kulantes Vorgehen seitens der Steuerbehörden angebracht, soweit das Gesetz es zulässt.

Aufgrund der neuen Transparenz der Vorschriften müssen die Deklarationspflichten ernst genommen werden. In der Wegleitung zum Ausfüllen des Lohnausweises wird darauf hingewiesen, dass, wer einen Lohnausweis nicht oder falsch ausfüllt, bestraft (Art. 127, 174 und 186 DBG; Art. 43, 55 und 59 StHG sowie Art. 251 StGB) und/oder haftbar (Art. 177 DBG; Art. 56 StHG) gemacht werden kann. Beim Lohnausweis handelt es sich um eine Urkunde.

Die Wegleitung zum Ausfüllen des Lohnausweises der Schweizerischen Steuerkonferenz (SSK) und der Eidgenössischen Steuerverwaltung (ESTV) beschreibt ausführlich die Formvorschriften. Mehrheitlich wurden die alten Vorschriften übernommen, mit Ausnahme der Gehaltsnebenleistungen (fringe benefits), die viel detaillierter und ausführlicher in verschiedenen Ziffern und Abschnitten zu deklarieren sind.

Als Gehaltsnebenleistungen gelten alle Leistungen, die nicht in Geldform ausgerichtet bzw. verbilligt oder gratis abgegeben werden. Sie sind zum Marktwert bzw. zum Verkehrswert zu bewerten. Eine Auswahl von Gehaltsnebenleistungen:

- Verpflegung und Unterkunft;

- Einkaufsbeiträge für die 2. Säule;

- Beteiligungsrechte;

- Aus- und Weiterbildungskosten;

- Geschäftsfahrzeug für private Zwecke: Die Berechnung des Wertes der privaten Nutzung von Geschäftsfahrzeugen (sogenannter Privatanteil) wurde einheitlich festgelegt. Übernimmt der Arbeitgeber sämtliche Kosten, so beträgt der zu deklarierende Betrag pro Monat 0,8\% des Kaufpreises exkl. MWSt, mindestens aber Fr. 150.- pro Monat (Stand September 2006). Bei Leasingfahrzeugen tritt an die Stelle des Kaufpreises der im Leasingvertrag festgehaltene Barkaufpreis des Fahrzeugs (exkl. MWSt). Beachten Sie aber, dass momentan bei der MWSt andere Berechnungswerte angewandt werden.

Aus Gründen der Praktikabilität müssen folgende Leistungen nicht deklariert werden:

- gratis abgegebene Halbtaxabonnemente der SBB;

- Reka-Check-Vergünstigungen bis Fr. 600.jährlich;

- übliche Weihnachts-, Geburtstags- und ähnliche Naturalgeschenke bis Fr. 500.- pro Ereignis;

- private Nutzung von Arbeitswerkzeugen (Handy, Computer usw.);

- Gratisparkplatz am Arbeitsort;

- Gutschriften von Flugmeilen. Sie sollen für geschäftliche Zwecke verwendet werden;

- Beiträge an Vereins- und Clubmitgliedschaften bis Fr. 1000.- im Einzelfall. Bei Beiträgen, die diesen Betrag übersteigen, ist der ganze Betrag anzugeben;

- Beiträge an Fachverbände unbeschränkt;

- Beiträge an Aus- und Weiterbildung, wenn diese direkt durch den Arbeitgeber bezahlt werden und Fr. 12000.- pro Jahr und Mitarbeiter nicht übersteigen;

- usw. 
Eine weitere wichtige Neuerung erfolgt im Bereich der Deklaration von Spesenzahlungen. Hier wird unterschieden zwischen effektiven Spesenvergütungen anhand von Belegen, pauschalen Spesenvergütungen, die für einen bestimmten Zeitabschnitt bezahlt werden, und Spesenvergütungen im Rahmen eines von den Steuerbehörden genehmigten Spesenreglements.

Das Spesenwesen ist deshalb so auszugestalten, dass das Deklarationsverfahren anschliessend einfach erfolgen kann. Es ist zu empfehlen, die Spesen in einem Spesenreglement festzuhalten. Das Reglement ist von den Steuerbehörden zu genehmigen. Der Vorteil genehmigter Spesenreglemente ist, dass das Unternehmen von der betragsmässigen Deklaration der effektiven Spesen dispensiert ist. Gerne unterstützen wir Sie beim Erstellen eines solchen und bei dessen Bewilligungsverfahren.
Wir empfehlen Ihnen, folgende Schritte vorzukehren, damit Sie keine negativen Überraschungen erleben:

- Einführen/Anpassen an einfache und leicht anzuhabende Lohn- und Gehaltsstrukturen;

- Vermeidung von Spesen mit Lohncharakter;

- Überprüfung/Anpassung/Einführung des Spesenreglements;

- Genehmigung des Spesenreglements;

- alle Lohnnebenleistungen klar definieren und schriftlich festhalten;

- allenfalls Anpassung der Arbeitsverträge;

- Anpassung der Informatik;

- Anpassung der Lohnstammdaten;

- Überprüfung der Auslagerung der Lohnbuchhaltung an eine Treuhandgesellschaft.

Gerne stehen Ihnen die Vertrauenspartner der FMH Treuhand Services für Fragen jederzeit zur Verfügung. 